\title{
Analysis and Preventive Measures on Bird Pest of Transmission Line
}

\author{
Bo Liu, Changxin Ge, Qiang Liu, Yi Wang, Xinnan Xie, Yanjun Pang, \\ Chenyang Liu, Liu Wang, Yanhai Huang \\ Fushun Power Supply Company, Liaoning Electric Power Company Limited, State Grid, China
}

\begin{abstract}
Keywords: Flashover, Bird Droppings Flashover, the Bird Thorn, Big Hat Insulate, Bird Shields
\end{abstract}
\begin{abstract}
Guano flashover belongs to a form of pollution flashover, whose process is complex. Bird droppings shows liquid and has a certain viscosity and high conductivity, especially the conductive rate of some wading bird droppings reaches $6000^{\sim} 8000 \mu \mathrm{s} / \mathrm{cm}$. This article expounds the guano flashover generally can be divided into the following four conditions, and analyzes the reason causing the guano flashover, examples and proposes the corresponding solution measures.
\end{abstract}

\section{Introduction}

Pollution flashover is short for filthy external insulation flashover of power transmission and transformation equipment. Pollution flashover is surface flashover under filthy conditions along the surface of insulator, which because the surface of the insulator is dirty and wet, under the effect of voltage occurring surface flashover phenomenon throughout the poles along the surface of insulator surface. The three elements of flashover are filth, damp and voltage[1].

Guano flashover belongs to a form of pollution flashover, whose process is complex. Bird droppings shows liquid and has a certain viscosity and high conductivity, especially the conductive rate of some wading bird droppings reaches $6000 \sim 8000 \mu \mathrm{s} / \mathrm{cm}$. Guano flashover generally can be divided into the following four conditions[6].

(1) When birds rest on tower cross arm at night, they digest the food they have ate during the day after midnight, then defecate down by standing on the cross arm. Sticky feces scatters from tower cross arm on the insulator string, and then flowing down the insulator string, which makes insulator umbrella skirt into short sub and reduces creep distance, resulting in the occurrence of flashover of insulator string. This kind of failure exists much, and the point of it is also very obvious. Generally, there are obvious guano droppings on the insulator, conductor and cross arm. There are flashover traces at the surface of the insulator, and marks of discharge on the wire, hardware and cross arm.

(2) Some larger birds defecated when they were flying over the suspension point of insulator string. Because the guano droppings is a kind of viscous liquid, which under the action of gravity acceleration, forms a slender next fall. The whereabouts of the first part is always falling faster than the back. Therefore, as the falling time lasts, the distance between the beginning and the end of bird droppings will be longer and longer. The slender falling of bird droppings has a high conductivity which makes serious distortion of the distribution of electric field around insulator string, and dielectric strength decline, resulting in air gap breakdown. Such failures are common, large areas of bird droppings can often be found on the fault point tower below the ground.

(3) Usually, birds stay between the cross arm, the quantity of their droppings is small, not directly causing the line tripping. However, days and months multiplying, the guano droppings unclean on the insulator and cross arm will gradually thicken, that flashover trip will happen in case of rain, drizzle or fog. When this kind of failure finished, the ground below failure tower will have no bird droppings or fresh bird droppings.

(4) Birds droppings falls continuously, which also shrinks the ground distance of charged conductor constantly. When combined discharge gap reaches to a certain extent, conductor will discharge to ground wire by bird droppings. Under the effect of air convection, the whereabouts movement speed and trajectory of bird droppings are also changing. The endpoint of their dung bunch and the corresponding point under the charged body are erratic. Therefore, wire discharge traces is not usually on the corresponding points of bird droppings' vertical drop, but deviates some distance by the effect of air convection. When the wire discharging through the discharge channel to 
the ground, the bird droppings is intermittent, which formed many combined gap to produce electric arc, a few red arc heat made its gasification or carbonized. So it's difficult to find the obvious discharge traces from the tower component of the ground.

\section{Accident Cases}

On August 21, 2013, eight past five, $220 \mathrm{kV}$ lines C phases in the single-phase grounding fault occurred, reclosing device, then the line overlap succeed. According to the fault location, sending work areas organized forces immediately to go up to tower to check the fault on the red line in the 78\# 99\# tower $10.5 \mathrm{~km}$ line. Inspectors found a large number of bird droppings on tower 84\# and tower 85\#. Because the bird droppings pollution accidents have happened before replacing the composite insulator of tower 85\#. Inspectors examined the two base towers carefully, and then they found that there were bright spots of $700 \mathrm{~mm}$ length on the surface of $84 \# \mathrm{C}$ phase large lateral wire clamp external conductor. Then the charged class had the equipotential review, they found that there was an obvious burn upper end of composite insulator mandrel $0.7 \mathrm{~m}$, and the burning point was over the edge of upper and lower two pieces of umbrella skirt, slightly yellowish caramel color. There was a size of 1.5 burning on the upper steel cap. Therefore, they confirmed that 84\# was the failure point of this failure.

\section{Failure Analysis}

(1)The red lines of 78\# 99\# are along the river areas. There are often a large group of birds, herons, magpies, crows, etc to nest or rest on the tower. The habit of these birds' feeding is complex, which they forage for food during the day and then have a rest at night. After digesting overnight, early in the morning is the time that they shat relatively concentrated with the largest number of droppings, which is thin and sticky, with a string each time. We can always find more than $2 \mathrm{~m}$ long dung on the insulator string and the tower material. This is the main condition that the conductor forming combination to put lighting collaterals.

(2)Geographically river geomorphic troposphere air forms the irregular discharge channel. By the effect of convection air, bird droppings shows non linear shaped or nonlinear free falling, which forms a liner channel off and on because of different concentration and viscosity differences to relatively fall. This leads to the combination of irregular discharge gap.

(3) In the river and low-lying areas, the humidity of air is the largest at $4 \sim 7$ o'clock in the morning, which often forms the morning fog, in common conditions under the influence of humidity and morning fog that leads to big air suspended particles of dust and high density. This forms the favorable conditions of gas discharge breakdown. General line to ground fault usually occurs in this period of time (except external force such as lighting, mechanical failure).

\section{Measures to Prevent Bird Pest}

Due to the red line of the sound in the $220 \mathrm{kV}$ accident is quite frequent; it has already become a factor to threaten the red line safe operation. In 2014 and 2015, relevant persons were organized to observe on-site, they found that there was a kind of bird perching on the cement cross arm at 9 o'clock every night, whose body was very big, and the fault time mostly occurred from 9:30 in the evening to 6:30 in the morning the next day, the main reason for the line accident line tripping caused by bird droppings.

Install the bird shields.

Install the bird shields in the $110 \mathrm{kV}$ and $500 \mathrm{Kv}$ line insulator string. Bird shields are made up of plastic insulation materials, which have the center hole and their edges have a fan gap circle of plastic, just like the shape of straw hat after installed. The edge of bird shields is $70 \mathrm{~mm}$ bigger than the skirt edge of insulator porcelain, when the birds defecate on the insulator string; the bird droppings will pull on the bird shields, thus avoiding the insulator flashover occurred. Use of the opportunity of outage maintenance, in each of $110 \mathrm{kV}$ insulator add a piece, installed in the first 
piece of insulator on cross arm, $500 \mathrm{kV}$ line insulator string can be installed two or three tablets, namely by the first piece of cross arm, put one to two among insulator string.
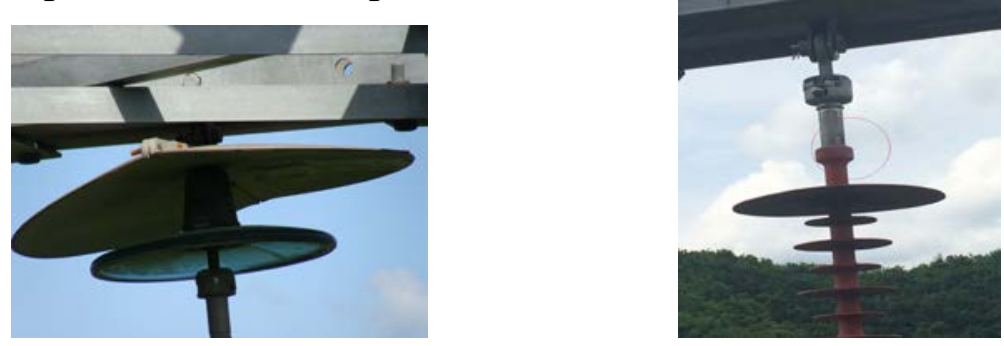

\section{The adoption of big hat insulator.}

Tripping caused by bird droppings flashover, less points but wide surface makes it not fixed. Bird droppings flashover accident occurs mostly at 4 5 o' clock in the morning, most big birds stand on the opposite ends of the cross arm, bird droppings falls along the edge of the insulator that leads to the cross arm discharge. In the upper insulator string, adding up to a large hat insulator should be able to have the effect to prevent guano whereabouts. However, we consider that adding insulator need to increase a lot of work, so the synthesis of silicone rubber paste way at the top of insulator can also help to prevent guano whereabouts, anti-icing shim, anti-fog pine flashover.

So it's safe for maintenance personnel to use "synthetic insulation big umbrella" in the past. The diameter of the big umbrella is $100 \mathrm{~mm}$ larger than the edge of the anti-pollution insulator; which is $150 \mathrm{~mm}$ larger than the edge of the composite insulator. It should be said that the use of this kind of big umbrella has played a considerable role.
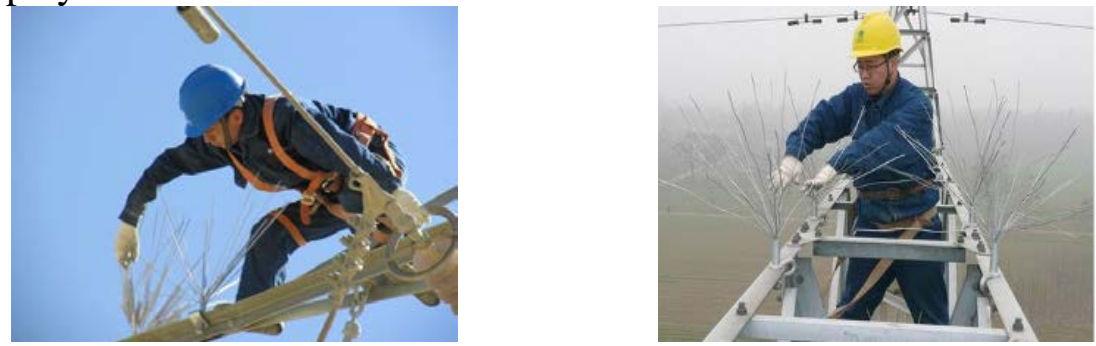

\section{Install repelling thorn.}

Installing repelling thorn above cross arm of the tower suspension insulator string, the repelling thorn is made up of steel strand GJ-70, whose length is :110kV line $350 \sim 450 \mathrm{~mm}, 500 \mathrm{kV}$ line $500 \mathrm{~mm} \sim 550 \mathrm{~mm}$. The steel strand board should be installed at the end of the U-shaped card, one side scatters radiated that there is a thimble screw under the U-shaped card, card installed on the cross arm angle, with a thimble screw tight.

$66 \mathrm{kV}$ of the sound of the fault phase occurs in the edge, $220 \mathrm{kV}$ of the sound of the fault is in phase, which is because the structure of the phase cross arm in $220 \mathrm{kV}$ tower is applicable to the bird nest. One repelling thorn is installed in each phase of $66 \mathrm{kV}$ line, $220 \mathrm{kV}$ line due to cross arm wide, two are installed in each phase, and repelling thorn can effectively block insulator string. Repelling thorn can effectively prevent guano stay on the tower, which is a good method that simple, easy to install. However, it has influence to the maintenance staff to do assignment on the pole, due to personnel movement, even inadvertently be stabbed by repelling thorn, so maintenance staff should be careful when they finish assignment on the pole.
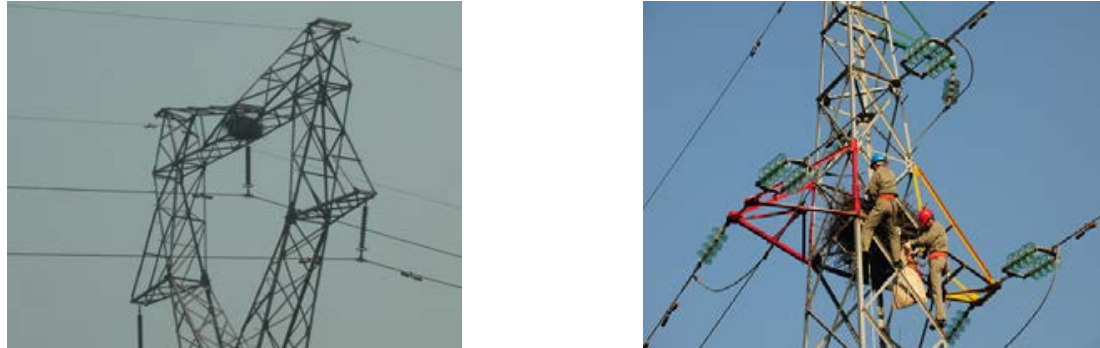

Dismantle the bird's nest in time.

When we find the bird's nest on the tower, we should report it in time, and remove it timely. 


\section{Using improved rotation device of chasing bird.}

The rotation device of chasing bird has been developed, which is composed of a rotation axis and a few simple framework. The shaft sleeve rotation using bearing, usually turn the use of wind, birds dare not be near. When a bird falls on the frame, the device is running and makes the bird be frightened that it can't rest on the frame. Bird pest accident has not happened since the device is installed. Later we found that there was a bird pest accident in three $110 \mathrm{kV}$ line $111 \#$ rod, and on-site inspection found that the gear rotational part had been basically rust duo to the snow and rain, which lost the function of chasing bird. We need change the bearing to maintain original performance, but replacing the bearing will bring a lot maintenance work. Later the GJ-35 steel wire was fixed on the top of the dry tower, whose length was $1.5 \sim 2.5 \mathrm{~m}$, then the steel strand was to be put to pry, forming a large radius of protection. When the bird falls on each strand wire, it can't fell on the dry tower, which because gravity makes the steel strand of jitter. And the device and lightning eliminator are separated that not affect the performance of the lightning eliminator, and can also prevent the harm accident effectively. When installing the device to prevent birds, we also improve the mode of the combination of the insulator string by changing the original way of $7 \times \mathrm{XWP}-7$ or $8 \times \mathrm{XWP}-7$ to $1 \times \mathrm{XMP}-7+7 \times \mathrm{XWP}-7$, so as to prevent bird pest through the first piece of straw hat type of porcelain insulator.

Through the measures above, bird pest of $110 \mathrm{kV}$ lines are greatly reduced, and general accident and the first grand obstacles have not happened for several years. At the same time, line number of annual trip is also greatly reduced.

\section{Conclusions}

The bird pest accident of the transmission line is on the rise, the important reasons are the promulgation and implementation of the national wild animal protection law and the forestry law, all levels of government public opinion publicity, the general increasing awareness of wildlife protection by national, the reducing phenomenon of excessive hunting wild animals, in addition, the strengthening conservation work of soil and water, the improvement of the natural environment, which create conditions for the reproduction of wildlife. These all put forward a new problem for transmission line maintenance work.

According to the analysis of operation condition, several line faults have not been found, which may also be the reason by the bird pest accident. It's because the weather was fine when the accident occurred that ruled out the possibility of thunder pest, the external force damage and scissors crossing fault. Due to the long line, checking line is not timely, and the rain will wash the bird droppings' trails cleanly after raining. Although we do not find the fault point by checking the line again and again, the traces of flashover on the insulator can also be found when repairing the failure line power, so this type of fault can also be caused by bird droppings.

\section{References}

[1] Yu Huayu Xu Wencheng Shen Gang etc. High voltage electrical equipment antifouling flash and charged cleaning technology. China electric power press, 2006.6

[2] Liu Zhenya. Uhv ac transmission system external insulation. China electric power press. 2008.6

[3] Wang Changchang Li Fuqi Gao Shengyou etc. Online monitoring and fault diagnosis of power equipment. Tsinghua university press. 2006.3

[4] Hebei province. The electric power research institute electricity antifouling flash technology quiz. China electric power press. 2009.9

[5] Qiu Zhixian. High voltage composite insulator and its application. China electric power press. 2006.1

[6] Electric power research institute of Hebei Province. The question and answer of grid antifouling flash technology. China electric power press. 2009 